\section{Spatial determinants of depth perception in two-dot motion patterns*}

\author{
ERIK BÖRJESSON and CLAES VON HOFSTEN† \\ University of Uppsala, Uppsala, Sweden
}

The aim of the present study was to identify spatial properties of motion patterns yielding perceived depth. A model was proposed to describe the different motion patterns in terms of the common and the relative motions of the moving parts. Using motion patterns consisting of two dots moving in straight paths, it was found that there were three different types of relative motions yielding unambiguous percepts: (1) lack of relative motions was perceived as motion in a frontoparallel plane; (2) two-dimensional concurrent motions were perceived as translatory motion in depth; (3) nonconcurrent motions were perceived as rotation in depth. Only two-dimensional motion patterns yielded stable depth percepts. The utility of the proposed vector analysis was discussed, and further research suggested.

The human visual system provides specific percepts of a three-dimensional world, although the light entering the eye is extended in two spatial dimensions only. This fact is commonly accounted for in terms of a variety of binocular and monocular depth cues. Although motion parallax is often included in the list of depth cues, most studies have used static targets as stimuli, and the effects of different kinds of motions have been neglected.

However, another line of research has stressed the importance of motion patterns for depth perception (Wallach \& O'Connell, 1953; Johansson, 1964, 1971). The present investigation is closely related to this approach, and its general purpose was to develop a vector model for prediction of perceived motion from two-dot patterns.

\section{THE MODEL}

The eye never perceives the individual proximal motion paths of a group of elements but instead combines motion components to perceptual units. In this respect the visual system functions in analogy with a mathematical vector analysis of the proximal pattern (Johansson, $1950,1971)$. In other words, an individual motion within a motion pattern is represented by several component motions.

\footnotetext{
*The authors are indebted to Professor Gunnar Johansson for valuable discussions on this project and for his constructive criticism of the manuscript. The authors are also indebted to Dr. Gunnar Jansson for his valuable comments on the manuscript. The responsibility for this investigation is equally shared between the authors. This investigation was made possible by grants to Professor Johansson from the Swedish Council for Science Research and the Tricentennial Fund of Bank of Sweden.

†Address: Spepartment of Psychology, University of Uppsala, Svartbäcksgatan 10, S-753 20 UPPSALA, Sweden.
}

The different motion patterns used in this study will be described in terms of a set of component motion vectors in the frontoparallel plane. Such a vector analysis can be carried out in an indefinite number of ways unless one or several guiding principles are introduced. It has been demonstrated repeatedly that the eye extracts that motion which is common to the moving parts (Wertheimer, 1923; Duncker, 1929; Johansson, 1950). Therefore the present model states that each individual motion is divided into (1) a common component motion, and (2) a relative component motion. The common component

motion is equal in magnitude and direction for all individual motions, and the relative component motion is determined by the individual motion and the common motion. The magnitude and direction of the common component motion is so determined that the sum of the relative residual motion vectors equals zero. In Column 1 in Fig. 1, three different two-dot motion patterns are shown. The arrows indicate the magnitude and direction of the movement in the frontoparallel plane for the dots which are moving back and forth in their respective paths. In Columns 2 and 3 , two phases of the cyclic motion are described according to the model. A common component motion is extracted from the two motions of each pattern. The magnitude and the direction of the common motion for each pattern are shown by the arrows outside the rectangular frames. (These frames were not shown in the experiments, but only the two moving dots.) The arrows inside the frames show the relative residual motions, if any. Note that the sum of relative motion vectors within each frame equals zero, i.e., the two relative motion vectors are of the same magnitude, are parallel, and point in opposite directions.

The motion patterns in Fig. 1 illustrate the three possible cases of relative motions of two dots moving with constant velocity in straight paths. In Pattern $A$, the relative

II

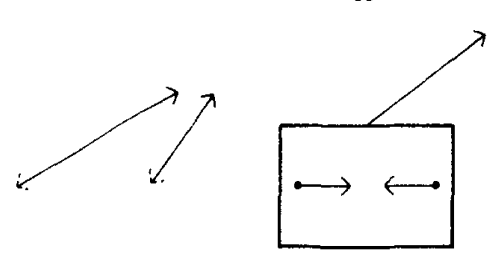

A
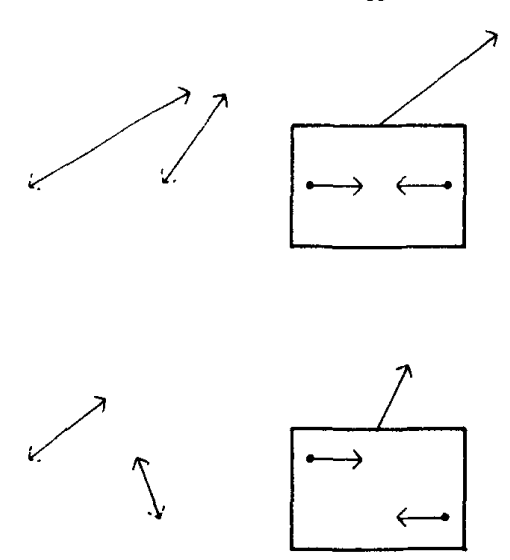

B
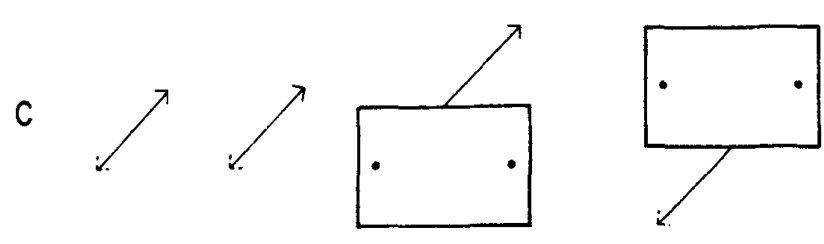

Fig. 1. Different cases of relative motions. In Column 1 the actual motion paths are shown and in Columns 2 and 3 two phases of the motion in terms of the vector analysis proposed. 
B
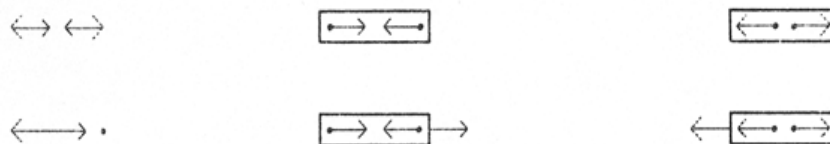

C

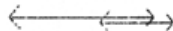

$0 \quad$

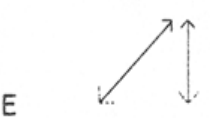

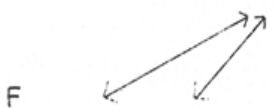

$\uparrow$
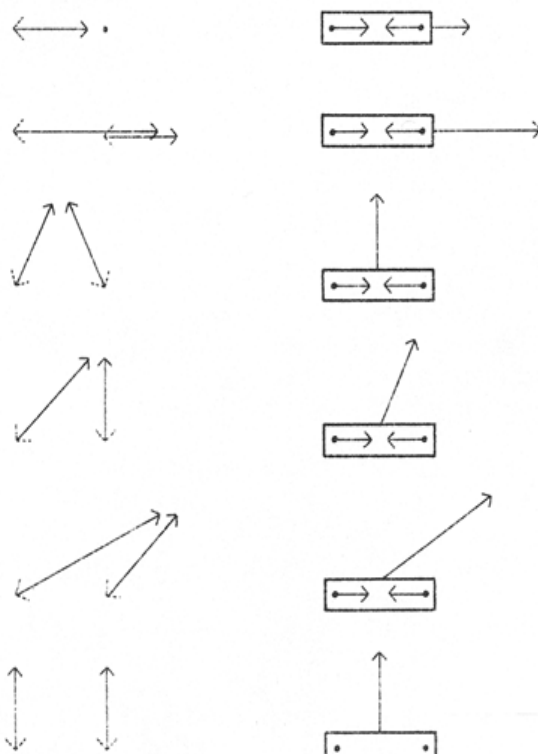
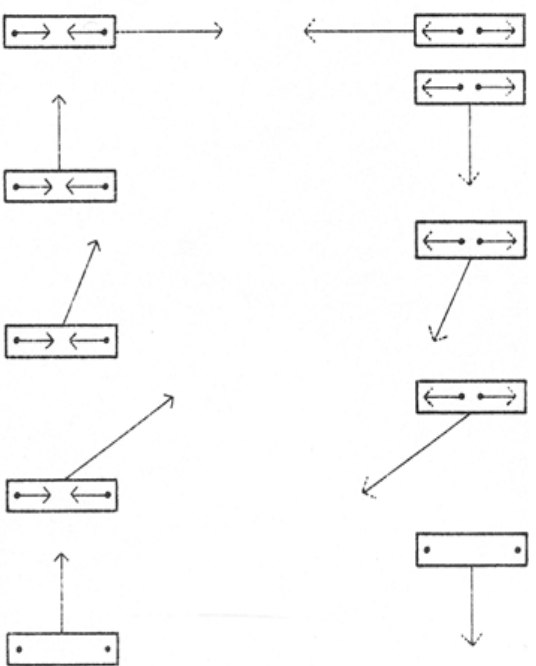

Fig. 2. Motion patterns used in Experiment 1. In Column 1 the actual motion paths are shown and in Columns 2 and 3 two phases of the motion in terms of the vector analysis proposed.

motions are aligned, i.e., the dots would meet in a common point in the frontoparallel plane if their motions toward each other continued. This type of motion has been extensively discussed by Johansson (1971) and is called concurrent motion. In Pattern $B$, the relative motions are not aligned. This type of relative motions will be called nonconcurrent motions. Finally, Pattern $\mathrm{C}$ illustrates the case with no relative motions.

The purpose of the following experiments was to study the effect of common and relative motions on perceived motion.

\section{EXPERIMENT 1}

In Experiment 1 concurrent motions with different common motions were studied. Wallach and O'Connell (1953) found with a shadow of a rotating $T$-figure that the kinetic depth effect was very weak. Since the T-figure is very similar to concurrent motions of two dots without a common motion, it is predicted that this pattern (Pattern A in Fig. 2) will not yield stable depth percepts.

The addition to concurrent motions of a common motion vector parallel to the relative motions does not change the dimensionality of the motion pattern; it is still one-dimensional (Patterns B and C in Fig. 2). When the common motion vector added is not parallel to the relative motions, the change becomes two-dimensional (Patterns D, E, and F in Fig. 2). Wallach and O'Connell (1953) and Johansson (1964) concluded that two-dimensional changes in the proximal stimulus were essential for evoking perceived depth. However, they did not use one-dimensional changes in which common motions were present. The addition of a common motion vector always implied a two-dimensional change. The purpose of Experiment 1 was to study whether it was the common motion vector or the dimensionality of proximal change that evoked stable depth percepts. Further, a pattern with no relative motions was studied.

\section{Apparatus}

A digital computer (Linc-8) was programmed to generate the desired stimulus patterns. The analog output of the computer was fed into an oscilloscope (Tectronix 565) which displayed the two dots by means of an optical device onto a translucent screen. A collimator lens was placed in front of the screen in order to minimize cues of two-dimensionality. This apparatus was used in all the experiments to be reported.

\section{Stimuli}

The stimulus patterns in Experiment 1 consisted of two dots, one or both of which were moving. The paths of these dots for some of the stimulus patterns are presented in Fig. 2. 'The actual motion paths are shown in Column 1 in Fig. 2. Columns 2 and 3 show two phases of the motions in terms of the vector analysis proposed.

Pattern A consists of two motion vectors of equal size but opposite directions. A common motion is added in Patterns $B$ and C. Since this common motion has the same direction as the left dot, the change is still one-dimensional. In Patterns D, E, and $F$, however, the addition of the common vector makes the proximal stimuli two-dimensional. Finally, Pattern G illustrates the case with no relative motion and a common motion vector perpendicular to the dimension of the extension of the two dots. In addition to these seven stimulus patterns, another four were included in Experiment 1. These patterns corresponded to Patterns A, B, D, and $E$ with the exception that the horizontal distances were doubled and the velocity of the moving dots changed accordingly since exposure time was held constant for all stimulus patterns.

\section{Viewing Conditions}

Each $S$ was seated with his head about $20 \mathrm{~cm}$ in front of the collimator lens. The S looked binocularly through the lens at the moving dots projected onto the translucent screen. Since the collimator lens gave parallel light rays, binocular depth cues were strongly reduced. The different motion patterns were presented in the following way. The two dots appeared simultaneously at corresponding ends of their motion paths and began immediately to move along their paths. The motion lasted for $3.4 \mathrm{sec}$, and then the dots disappeared. After a pause of $1.9 \mathrm{sec}$, the dots started from the place where they had disappeared and, thus, described their respective paths in opposite direction. This motion also lasted for $3.4 \mathrm{sec}$, and after another pause of $1.9 \mathrm{sec}$, during which the dots were not presented, the cycle continued. The greatest distance between the dots of Patterns A-F in Fig. 2 subtended a visual angle of $2.3 \mathrm{deg}$, and the smallest distance a visual angle of $0.4 \mathrm{deg}$. The horizontal extension of the common motion for Patterns B and E was 0.9 deg and for Patterns $\mathrm{C}$ and F, $2.8 \mathrm{deg}$. These visual angles were doubled for four other patterns corresponding to Patterns A, $B, D$, and $E$. In the two-dimensional patterns, the displacement in the vertical dimension always subtended a visual angle of $1.9 \mathrm{deg}$. The distance between the dots in Pattern G corresponded to a visual angle of $2.3 \mathrm{deg}$. 
Procedure

Before the experiment, the Ss were told that two moving dots would be presented. It was suggested that these dots might appear to move in a plane perpendicular to the line of sight, in different circular paths, describe translatory motion in depth, or any combination of these alternatives. The Ss were asked to report verbally what they perceived, or, when uncertain as to how to express the perceived event verbally, to show the motions of the dots with their hands. In the case of perceived translatory motion in depth, the Ss were further requested to rate the strength of this impression in a scale with four categories: (1) very weak, (2) rather weak, (3) rather strong, and (4) inevitable. Johansson (1950) has made clear that the perception of motion patterns is influenced by the fixation behavior of the S. A fixated dot tends to be perceived as a frame of reference for the motions of the other dots. The Ss were therefore asked not to fixate on a particular dot. Before each presentation, the room was darkened and the stimulus pattern appeared in a random phase of its cyclic course. Inspection time was free. Each stimulus pattern was presented twice in randomized order, giving 25 presentations including three test trials before the experiment.

\section{Subjects}

Ten Ss served in Experiment 1.

Results

The reports were classified in the following four categories: (1) Translatory motion in a frontoparallel plane (PLANE). (2) Translatory motion in depth (DEPTH). (3) Motion in circular or nearly circular paths with the dots at different distances from the eye. This perception was often described as rotation in depth of a rod of which only the end points were seen (ROTATION). (4) A simultaneous combination of Categories 2 and 3 (DEPTH + ROTATION).

The four categories are illustrated in Fig. 3. The arrows on the picture plane indicate the proximal motion pattern, and the pairs of arrows behind the picture plane indicate perceived motions in space according to

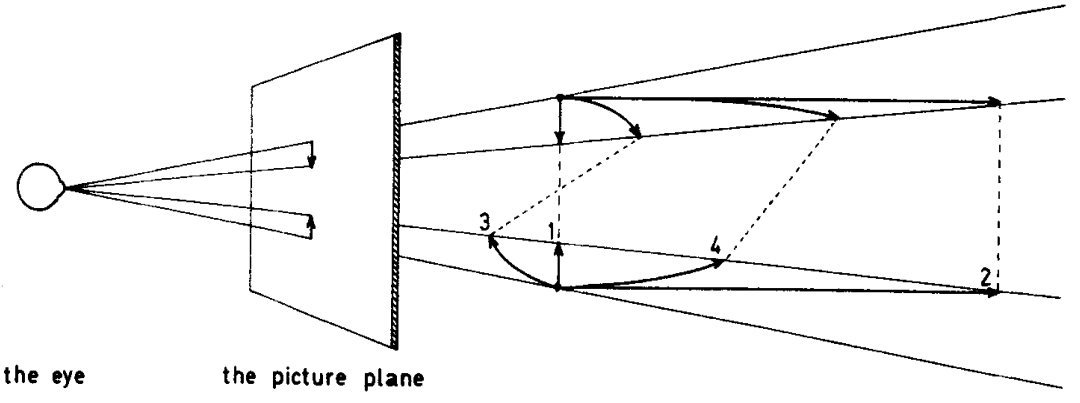

Fig. 3. Different response categories of perceived motions. The numbers refer to the following categories: 1, PLANE; 2, DEPTH; 3, ROTATION; and 4, DEPTH + ROTATION.

Categories 1, 2, 3, and 4. The dotted lines between the arrows were not perceived but are introduced in Fig. 3 to show which arrows belong together. In Category 1 the whole change of distance between the dots in the proximal motion pattern is perceived as a change of distance between the dots in a frontoparallel plane. In Categories 2, 3, and 4 the change of proximal distance between the dots is perceived as motion in depth.

Since the two versions of Patterns $A, B, D$, and E yielded about the same results, these are combined in Table 1. Table 1 shows that there is no difference between Pattern A, with no common motion vector on the one hand, and Patterns $B$ and $C$, with a common motion vector in the same dimension as the relative change of the dots on the other hand. Patterns A, B, and $C$, which changed in one dimension, received responses in all of the response categories. However, Patterns D, E, and F, with two-dimensional change almost always yielded percepts of translatory motion in depth. When the dots moved toward each other they appeared to recede from the $S$, and when the dots moved away from each other they appeared to approach the $S$. The effect of the number of dimensions of change on frequencies of perceived translatory motion in depth (Categories DEPTH and DEPTH + ROTATION) was tested with the Wilcoxon matched-pairs signed-ranks test (Siegel, 1956). It was found that Patterns $D, E$, and $F$ (two-dimensional change) gave significantly more percepts of translatory motion in depth than
Patterns A, B, and C $(\mathrm{p}<.01$, one-tailed testing for $\mathrm{T}=0$ and $\mathrm{N}=7$ ). It was further found that the reported strength of the impression of the translatory motion in depth was greater for Patterns D, E, and F than for Patterns A, B, and C. Out of 62 reports of perceived translatory motion in depth for Patterns A, B, and C, $19.5 \%$ were judged "inevitable," $29 \%$ "rather strong," $32 \%$ "rather weak," and $19.5 \%$ "very weak." Corresponding values of the 98 reports of translatory motion in depth for Patterns D, E, and F were $59 \%, 25 \%$, $15 \%$, and $1 \%$. Pattern G, with no relative change of the two dots, was always perceived as moving in the frontoparallel plane.

\section{Conclusion}

To perceive motion in depth there must be a relative motion of the moving parts. If the proximal distance between the moving parts is constant, no motion in depth is perceived, but only motion in a frontoparallel plane. This conclusion is further supported by an experiment (Börjesson \& von Hofsten, 1971) using two-dot patterns without relative motions but having a common motion in the dimension of extension of the two dots.

Two dots moving with the same velocity toward or away from a coinciding point in one dimension yield a variety of percepts. Apparently this situation contains too little information for the visual system. The addition of a common motion vector in the same dimension as the relative change of the dots is not utilized by
Table 1

Frequencies of Different Kinds of Perceived Motion in Experiment 1

\begin{tabular}{lrrrrrrr}
\hline & \multicolumn{7}{c}{$\begin{array}{c}\text { Stimulus } \\
\text { Response }\end{array}$} \\
\cline { 2 - 8 } \multicolumn{1}{c}{ Category } & A & B & C & D & E & F & G \\
\hline Depth & 17 & 16 & 10 & 36 & 31 & 18 & 0 \\
Depth + Rotation & 5 & 10 & 4 & 4 & 8 & 1 & 0 \\
Rotation & 12 & 9 & 2 & 0 & 0 & 1 & 0 \\
Plane & 6 & 6 & 4 & 0 & 1 & 0 & 20 \\
\hline
\end{tabular}

Table 2

Frequencies of Different Kinds of Perceived Motion in Experiment 2

\begin{tabular}{lrlllll}
\hline \multirow{2}{*}{$\begin{array}{c}\text { Response } \\
\text { Category }\end{array}$} & A & B & C & D & E & F \\
\hline Depth & 0 & 0 & 0 & 0 & 0 & 0 \\
Depth + Rotation & 0 & 0 & 0 & 3 & 5 & 4 \\
Rotation & 10 & 8 & 8 & 6 & 4 & 6 \\
Plane & 0 & 2 & 2 & 1 & 1 & 0 \\
\hline
\end{tabular}


A

$\stackrel{\leftrightarrow}{\leftrightarrow}$

B

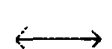

C

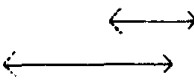

$0 \nearrow$<smiles></smiles>

$\mathrm{F}$

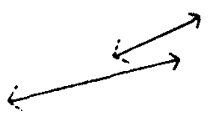

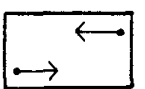
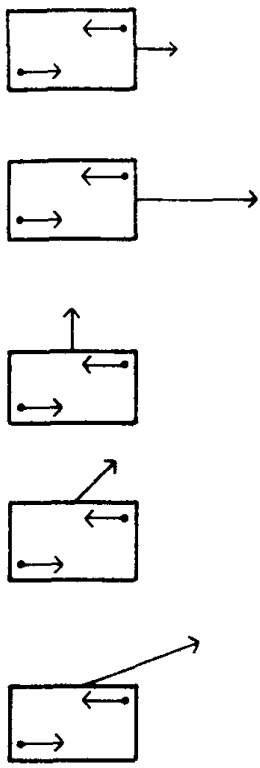

Fig. 4. Motion patterns used in Experiment 2. In Column 1 the actual motion paths are shown and in Columns 2 and 3 two phases of the motion in terms of the vector analysis proposed. For practical reasons, the vertical distances are halved relative to the horizontal distances in Fig. 4.

the visual system to determine perceived three-dimensionality. If, however, a common motion vector is added with a direction other than those of the relative motions of the dots, thus giving a two-dimensional change, translatory motion in depth is perceived. It is consequently concluded that concurrent motions changing in only one dimension contain too little information to produce predictable percepts of motion in depth, but that changes in two dimensions yield stable percepts of translatory motion in depth. The same conclusion was reached by Johansson (1964).

\section{EXPERIMENT 2}

In Experiment 2 nonconcurrent motions were studied, i.e., the relative motions were separated perpendicular to their directions. The influence of the separation of the relative motions was studied using different common motions.

Johansson and Jansson (1968), using an oscilloscope-generated line, the ends of which moved in parallel paths at different levels with opposite directions, found that $81 \%$ of the reports indicated perceived three-dimensional rotation with a fixed center. Since these stimulus patterns were similar to nonconcurrent motions of two dots, it is predicted that nonconcurrent motions will yield percepts of two dots at different distances moving in circular paths.

\section{Stimuli}

The stimuli were similar to those used in Experiment 1, consisting of two dots, of which one or both were moving. The motion paths of these dots are presented in Fig. 4. In Pattern $A$, there is no common motion vector. Patterns $B$ and $C$ have common motion vectors in the same direction as one of the relative motions, and Patterns D, E, and F have common vectors in other directions than those of the relative motions.

\section{Viewing Conditions}

The dots moved simultaneously back and forth in their respective paths. Presentation time for each motion was $3.0 \mathrm{sec}$ and the pause between, $1.9 \mathrm{sec}$. The greatest horizontal distance between the dots covered a visual angle of $2.3 \mathrm{deg}$ and the smallest, $0.4 \mathrm{deg}$. The vertical distance between the dots covered a visual angle of $1.8 \mathrm{deg}$, and the motion in the vertical dimension of Patterns $D, E$, and $F$ also covered a visual angle of $1.8 \mathrm{deg}$. The horizontal extension of the common motion was, for Patterns $B$ and E, 0.9 deg and for Patterns $C$ and F, 2.8 deg.

The same procedure was followed as in Experiment 1, with the exceptions that the Ss were not requested to rate the strength of perceived translatory motion in depth and that each stimulus was presented once in randomized order.

Ten Ss participated in Experiment 2.

Results and Conclusions

The reports were classified in the same four categories as in Experiment 1. The frequencies of these categories are presented in Table 2. Table 2 shows that almost all reports included some kind of perceived rotation in depth, sometimes accompanied by a translatory motion in depth. Thus, the stimulus information in this type of relative motions gives percepts different from those in concurrent motions. The prediction was confirmed for patterns with no common motion vector or a common motion vector in the same direction as one of the relative motions, i.e., Patterns $A, B$, and $C$ yielded motions of the dots at different distances from the eye in circular or nearly circular paths. Unexpectedly, however, in about half of the cases this motion was accompanied by a simultaneously perceived translatory motion in depth for the stimulus patterns which had common motion vectors in other directions than the relative motions, i.e., Patterns D, E, and F. Thus, these stimulus patterns were ambiguous with regard to perceived translatory motion in depth. The results are theoretically interesting in many respects. They imply that translatory motion in depth might be perceived in spite of the absence of concurrent motions in terms of the proposed vector analysis. Further, one wonders if there are certain motion patterns of two dots which unambiguously evoke perceived translatory motion in depth and a simultaneous rotation around a vertical axis. This possibility was not foreseen at the start of the study.

\section{EXPERIMENT}

Experiment 2 raised more questions than it answered. Experiment 3 was designed to investigate more closely under what conditions the different types of depth percepts occur. The preceding experiments made clear that the common motion vector not parallel to the relative motions and the separation of the relative motions perpendicularly to the relative motion paths are critical factors determining what kind of depth motion is perceived. The effects of these stimulus variables on perceived depth was further studied in Experiment 3. 


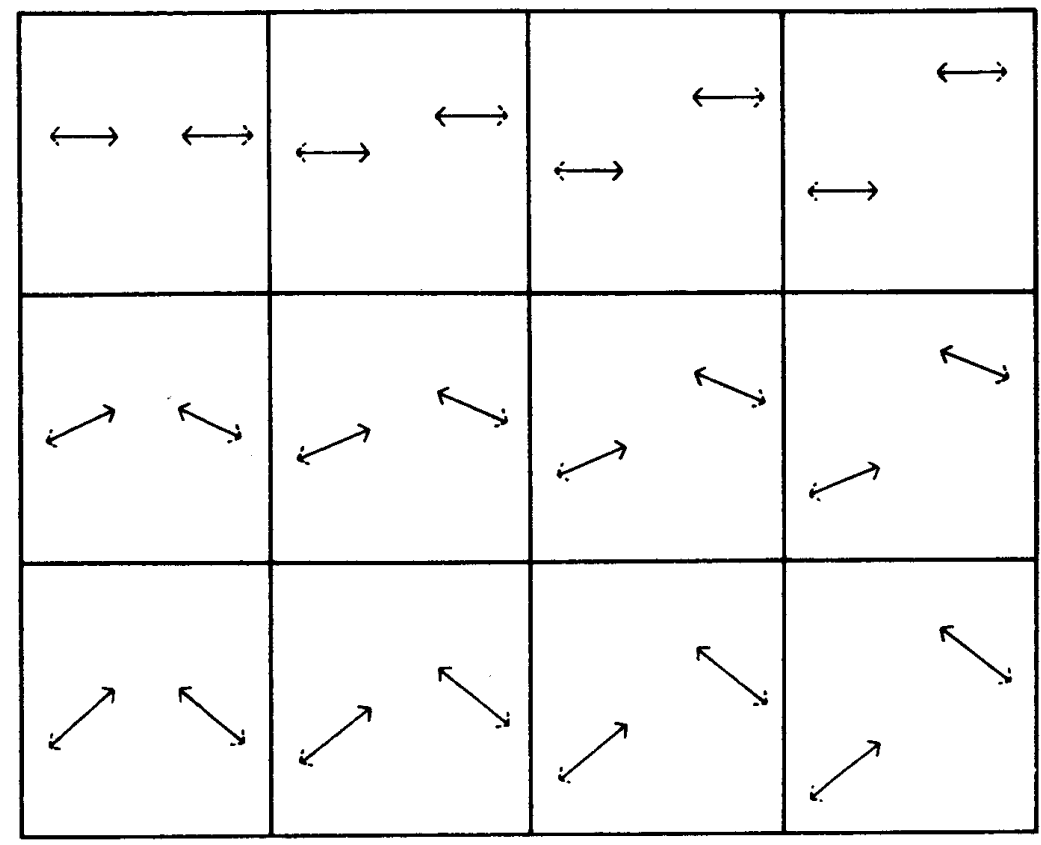

Fig. 5. Motion patterns used in Experiment 3.
Stimuli stimulus patterns consisted of two dots moving back and forth in their respective paths. These motion paths are shown in Fig. 5.

In the left column of Fig. 5, concurrent motions are presented. In the following columns with nonconcurrent motions, the relative motions are increasingly separated vertically. These separations correspond to visual angles of $0.7,1.4$, and $2.3 \mathrm{deg}$, respectively. The rows differ with regard to the magnitude of the common motion perpendicular to the relative motions. In the middle row, the common vector covers a vertical visual angle of $0.54 \mathrm{deg}$ and in the lower, $1.1 \mathrm{deg}$. The greatest horizontal extension of the dots covered a visual angle of $4.3 \mathrm{deg}$ and the smallest, $1.4 \mathrm{deg}$. Each motion lasted for $3.8 \mathrm{sec}$, with a pause of $1.0 \mathrm{sec}$ at the turning points during which the dots were not presented. A complete motion cycle was thus completed in $\mathbf{9 . 6} \mathrm{sec}$ for each stimulus pattern.

\section{Procedure}

The same instruction was given as in Experiment 2. Inspection time was free. Each stimulus pattern was presented twice in randomized order.

\section{Subjects}

Ten Ss participated in Experiment 3.

Results

The reports were categorized in the
As in earlier experiments, the same four categories as in Experiment 2. The different kinds of perceived motion for the 12 stimulus patterns are presented in Table 3 . The size of the common vector and the motions have systematic effects on perceived motion. The greater the common motion, the greater is the number of reported translatory motions in depth, and the greate $r$ the vertical separation of the relative motion paths, the greater is the number of reported rotations in depth. Thus, with no vertical separation between the relative motion paths (concurrent motions) and a great common motion, translatory motion in depth is almost always perceived, and with a great vertical separation between the relative motion paths and no common motion, rotation in depth vertical separation of the relative is perceived. However, when both a common motion vector and a vertical separation of the relative motions are presented, the atimulus patterns are ambiquous for the eye. Rotation in depth or rotation with a simultaneous translation in depth is perceived. Spontaneous reports indicate that when a combination of translatory motion in depth and rotation was perceived, much rotation was accompanied by little translation in depth and vice versa.

\section{DISCUSSION}

The purpose of the present investigation was to identify spatial properties that evoke perceived depth in proximal motion patterns. In order to isolate the critical variables, it was deairable to use stimuli that were as simple as posaible. Therefore, the spatial changes were produced with two-dot motion patterns. Under certain conditions these very simple stimuli gave stable percepts. Figure 6 summarizes the main perceptual effects of the different cases of relative and common motions.

It can be seen from Fig. 6 that constancy of proximal distance between the parts of a motion pattern, i.e., lack of relative motions, brings unambiguous information to the visual system of motion in a frontoparalle plane (Cells 2 and 3 ).

Although relative motions are necessary for perceived motion in depth, they are not sufficient. Concurrent motions in one dimension are perceived in different ways, including motion in a frontoparallel plane irrespective of the presence of a common motion vector (Cells 4 and 5). Thus, one-dimensional motion patterns with concurrent motions do not give unambiguous percepts. However, by adding a dimension and making the stimulus pattern two-dimensional, it is possible to obtain information which is unambiguous for the visual system.
Table 3

Frequencies of Different Kinds of Perceived Motion in Experiment

\begin{tabular}{|c|c|c|c|c|c|}
\hline \multirow{2}{*}{$\begin{array}{l}\text { Size of Vertical } \\
\text { Common Motion } \\
\text { Vector (Degrees } \\
\text { of Visual Angle) }\end{array}$} & \multirow{2}{*}{$\begin{array}{r}\text { Response } \\
\text { Category }\end{array}$} & \multicolumn{4}{|c|}{$\begin{array}{c}\text { Vertical Separation of Relative } \\
\text { Motion Paths (Degrees of Visual Anqle) }\end{array}$} \\
\hline & & $0 \mathrm{Des}$ & $0.7 \mathrm{Deg}$ & $1.4 \mathrm{Deg}$ & 2.3 Des \\
\hline 0 Des & $\begin{array}{l}\text { Depth } \\
\text { Depth + Rotation } \\
\text { Rotation } \\
\text { Plane }\end{array}$ & $\begin{array}{l}4 \\
5 \\
7 \\
4\end{array}$ & $\begin{array}{r}2 \\
5 \\
13 \\
0\end{array}$ & $\begin{array}{r}2 \\
3 \\
18 \\
0\end{array}$ & $\begin{array}{r}0 \\
1 \\
18 \\
1\end{array}$ \\
\hline $0.54 \mathrm{Deg}$ & $\begin{array}{l}\text { Depth } \\
\text { Depth + Rotation } \\
\text { Rotation } \\
\text { Plane }\end{array}$ & $\begin{array}{r}11 \\
3 \\
2 \\
4\end{array}$ & $\begin{array}{l}4 \\
7 \\
8 \\
1\end{array}$ & $\begin{array}{r}0 \\
6 \\
13 \\
1\end{array}$ & $\begin{array}{r}0 \\
1 \\
19 \\
0\end{array}$ \\
\hline 1.10 Deg & $\begin{array}{l}\text { Depth } \\
\text { Depth + Rotation } \\
\text { Rotation } \\
\text { Plane }\end{array}$ & $\begin{array}{r}17 \\
3 \\
0 \\
0\end{array}$ & $\begin{array}{r}\mathbf{5} \\
10 \\
\mathbf{5} \\
\mathbf{0}\end{array}$ & $\begin{array}{r}1 \\
14 \\
5 \\
0\end{array}$ & $\begin{array}{r}0 \\
9 \\
11 \\
0\end{array}$ \\
\hline
\end{tabular}


Properties of common motion

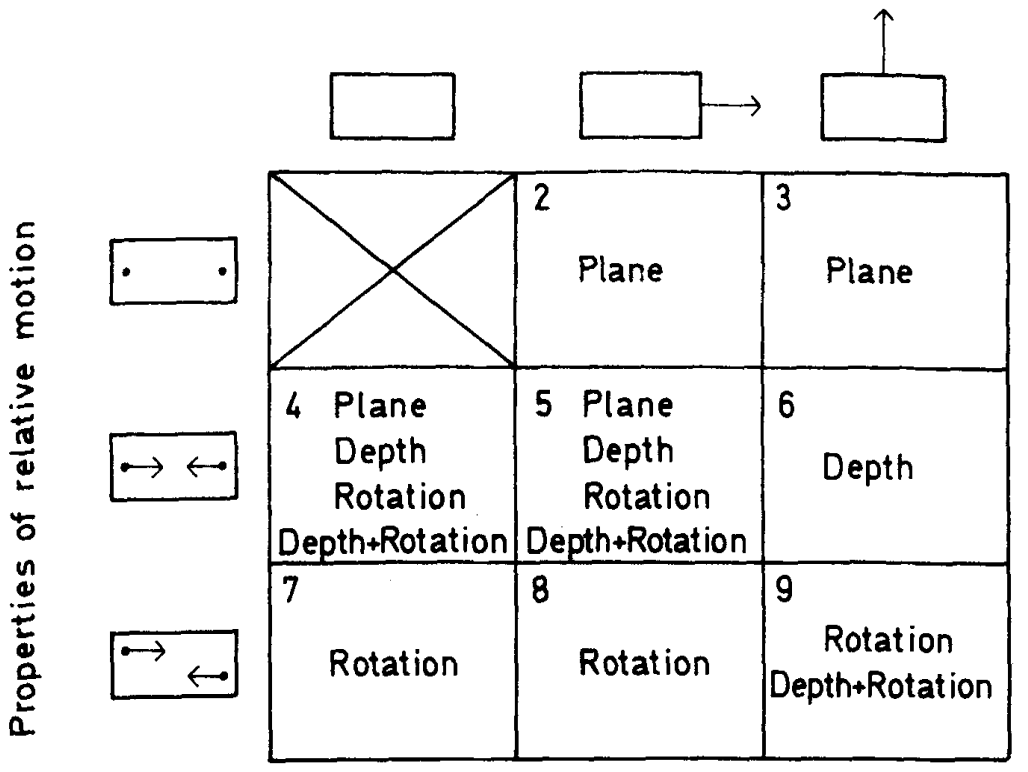

Fig. 6. Main percepts evoked by different combinations of common and relative motions.

There are two different and independent ways to make the stimulus pattern two-dimensional, each of which yields different but stable percepts. First, a common motion vector which is not parallel to the relative motions can be added, giving two-dimensional change of concurrent motions (Cell 6). This pattern is unambiguously perceived as a translatory motion in depth. Secondly, the two-dimensionality of the stimulus pattern can be achieved by separating the relative motions in the dimension perpendicular to the relative motions, i.e., nonconcurrent motions (Cells 7 and 8 ). These motion patterns bring unambiguous information to the eye of two dots moving in circular or circular-like paths at different distances from the eye. Often this percept was expressed as rotation in depth of a rod of which only the end points were seen.

In Cell 9 both a common motion not parallel to the relative motions and nonconcurrent motions are present. These motion patterns did not give stable percepts, but rotation was perceived with or without a simultaneous translation in depth. This result implies that the presence of concurrent motions in terms of the proposed vector analysis may not be a necessary condition for the perception of translatory motion in depth. However, it is possible that the eye has a tendency to extract concurrent motions in addition to the extraction of a common motion in this case.
Johansson (1964), using more complex stimuli, concluded that the visual system extracts concurrent motions which yield perceived translatory motion in depth and residual motion components which yield perceived rotation and changing shape. Experiment 3 showed that there was an increase in the tendency to extract concurrent motions and perceive translation in depth when the common motion was increased. An increase in the vertical separation of the relative motions favored perceived translatory motion in the frontoparallel plane and a simultaneous rotation in depth. In a pilot study it was tried to get stable percepts by introducing greater common motions and vertical separations between the relative motions. However, stable percepts were not found, but the results were similar to those in Experiment 3.

It is an outstanding result of this investigation that nearly all the perceived motions can be accounted for in terms of the minimum principle, e.g., a perceived constant distance between the dots. The perception of either rotation or translation in depth or a combination of these two categories can be interpreted in terms of an assumption of an object of constant physical size, the ends of which are defined by the points of light. The minimum principle cannot, however, predict under what conditions the different kinds of depth motion are perceived. In this respect, the proposed vector model is a successful tool in identifying relevant stimulus variables determining depth perception. Both the relative motions and the common motions are critical factors in the perception of depth motion.

As the relative motions have been produced only in the horizontal dimension, it can be argued that the results are limited to these conditions. Although control experiments are needed, it is highly probable that the direction of the relative motions is not a critical factor for the kind of event perceived. Johansson (1964) and Power (1968), working with changing stimulus patterns, found that the effects of the critical stimulus variables were not affected by the orientation of the stimulus patterns.

Any motion pattern, however complex, can be analyzed in terms of a set of relations between pairs of motion elements. In more complex stimulus patterns the relationships between stimulus variables and percepts found in this investigation may still be valid. On the other hand, other stimulus variables of higher order, not defined for two-dot patterns, may supply more convenient concepts for theorizing. The next natural step would be to study motion patterns of three and four moving dots.

\section{REFERENCES}

BÖRJESSON, E., \& VON HOFSTEN, C. Determinants of depth perception in motion patterns. Report from Psychology Laboratory, University of Uppsala, 1971 , No. 101.

DUNCKER, $\mathrm{K}$. Ùber induzierte Bewegung. Psychologische Forschung, 1929, 12, 180-259.

JOHANSSON, G. Configurations in event perception. Uppsala: Almqvist \& Wiksell, 1950.

JOHANSSON, G. Perception of motion and changing form. Scandinavian Journal of Psychology, 1964, 5, 181-208.

JOH ANSSON, G., \& JANSSON, G. Perceived rotary motion from changes in a straight line. Perception \& Psychophysics, 1968, 4, 165-170.

JOHANSSON, $G$. Visual motion perception: A model for visual motion and space perception from changing proximal stimulation. Report from Psychology Laboratory, University of Uppsala, 1971. No. 98.

POWER, R. P. Stimulus properties which reduce apparent reversal of rotating rectangular shapes. Journal of Experimental Psychology, 1967, 73, 595-599.

SIEGEL, S. Nonparametric statistics for the behavioral sciences. New York: McGraw-Hill, 1956.

WALLACH, H., \& O'CONNELL, D. N. The kinetic depth effect. Joumal of Experimental Psychology, 1953, 45, 205-217.

WERTHEIMER, M. Untersuchungen zur Lehre von der Gestalt. Psychologische Forschung, 1923, 4, 301-350.

(Accepted for publication September 20, 1971.) 